\title{
The Effect of Traverse Speed on Deposition Efficiency of Cold Sprayed Stellite 21
}

\section{Rajab Ali Seraj ${ }^{1}$, Amir Abdollah-zadeh ${ }^{1} *$, Sergi Dosta ${ }^{2, *}$, Horacio Canales ${ }^{2}$, Hamid Assadi ${ }^{3}$, Irene Garcia Cano ${ }^{2}$}

${ }^{1}$ Department of Materials Engineering, Tarbiat Modares University, Tehran, Iran

${ }^{2}$ Thermal Spray Center, Department of Materials Sciences and Engineering, Universitat de Barcelona, Barcelona, Spain

${ }^{3}$ Brunel Centre for Advanced Solidification Technology (BCAST), Brunel University London, UK.

* Corresponding authors.

Tel. /fax: +98 21 88005040. E-mail address: zadeh@modares.ac.ir (A. Abdollah-zadeh).

Tel./fax: +34 934034449. E-mail address: sdosta@cptub.eu (S. Dosta). 


\begin{abstract}
Cold spraying of Stellite 21 powder on low carbon steel is performed to investigate the effect of traverse speed on the deposition efficiency (DE) of high-temperature alloys. Based on the simulation of particles' impact temperature and velocity, the initial experiments are performed at different gas pressures (32 and 40bar), temperatures (800 and $\left.730^{\circ} \mathrm{C}\right)$, and stand-off distances $(10,25$, and $40 \mathrm{~mm})$ at a constant traverse speed of $20 \mathrm{~mm} \cdot \mathrm{s}^{-1}$. The experiments showed that high pressure, temperature, and short stand-off distance are preferred. The wipe test results indicated a potential of high DE at lower deposition flux over the substrate surface area at the first layer deposition. Thus, new tests are carried out at different traverse speeds ranging from 20 to $400 \mathrm{~mm} . \mathrm{s}^{-1}$ to adjust the deposition flux over the surface area of the substrate. The effect of traverse speed on DE and coating characteristics such as porosity and microhardness is studied as well. The results showed that the lower DE at lower traverse speed is related to the erosion of bonded particles due to the subsequent particles' impact. By increasing the traverse speed from 20 to $300 \mathrm{~mm} \cdot \mathrm{s}^{-1}$, the DE increased more than twice. Induction time extension at higher traverse speed led to lower DE at $400 \mathrm{~mm} \cdot \mathrm{s}^{-1}$.
\end{abstract}

\title{
Keywords:
}

Cold spray, High-temperature alloy, Stellite, Erosion phenomenon, Traverse speed, Deposition efficiency. 


\section{Introduction}

Stellite alloys are a series of cobalt-chromium alloys designed for high temperature, wear, and corrosion resistance applications, which are demanded by different industries. The exceptional galling resistance of Stellite alloys makes them an excellent choice for deposition on the parts of high-pressure gate valves [1-3]. The limitations of a conventional deposition process such as dilution, tensile residual stress, pores, and cracking have motivated researchers to use innovative deposition processes such as cold spray (CS) [3-10].

CS is a solid-state deposition process in which the metallic, ceramic, or composite powders are accelerating to a high velocity toward a substrate. Using optimized process parameters, a compacted coating could be achieved [11]. The absence of oxide layer and also compression residual stress in the optimized cold sprayed coatings make them an excellent choice for deposition of sensitive materials $[12,13]$. The feedstock powder is injected into a hot (max. $0.8 \mathrm{~T}_{\mathrm{m}}$ melting temperature of feedstock material) pressurized gas (e.g., $\mathrm{He}, \mathrm{N}_{2}$, and Air) and then passed through a de-Laval nozzle [14]. The gas pressure decreases remarkably upon leaving the nozzle throat due to rapid expansion. So, the thermal energy of the gas is transformed into kinetic energy and the gas velocity increases quickly $[15,16]$. The very short exposure of the injected particles in the hot gas stream (max. 3ms) and also decreasing the gas temperature in the nozzle throat section lead to preserving the initial microstructure of the powders, avoiding oxidation in the feedstock and undesirable phases in the coating [11]. Costeffectiveness, a relatively high deposition rate, and low temperature of the gas make CS more favorable than the conventional thermal spray and supersonic laser deposition methods for industrial applications [16]. In this regard, investigations are highly focused on the deposition of either high strength alloys or metal matrix composites $[1,11,17,18]$. 
Despite the exceptional characteristics of cold sprayed coatings, the deposition of less ductile materials such as cobalt-based alloys by CS is a challenging issue. Parameter selection complexity and low DE of high-temperature alloys are the main issues for deposition of these materials by CS. The higher the strength of these alloys, the lower deformability of their particles would be. The deformation behavior of the particles is affected by the impact velocity and temperature [19]. Moreover, the particles' impact temperature and velocity are affected by the CS parameters. The effect of process parameters consisting of process gas type, temperature, pressure, and substrate hardness, temperature, and roughness on the coatings' properties have been investigated by different authors $\left.\left[r_{-} Y_{-}\right)^{9}\right]$. Cinca et al. $[1,23$, 24] deposited Stellite 6 alloy on a steel substrate by the CS. Although they used an optimized value of gas temperature, pressure, and stand-off distance to achieve a dense and adherent coating, the reported DE for the selected parameters was about 20\% [24]. In addition, various traverse speeds have been used by some authors for high-strength alloy deposition [25-27]. Richer et al. [25] suggested that, by using a low traverse speed (The exact value of traverse speed has not been reported), a CoNiCrAlY layer with lower amount of porosity and higher mechanical interlocking could be achieved. However, the effect of low traverse speed on DE has not been considered by them. Varadaraajan and Mohanty [26] studied the effect of nozzle traverse speed ranging from 1.5 to $6 \mathrm{~mm} . \mathrm{s}^{-1}$ on the deposition rate of AISI 316 stainless steel powder. Kotoban et al. [27] investigated the effect of traverse speed ranging from 5 to $80 \mathrm{~mm} . \mathrm{s}^{-}$ ${ }^{1}$ on the DE of multi-pass single track deposition of SS316L. They showed that at lower traverse speed, the thickness of deposited layer increased and the impact angle of the particles (the angle between the particles' trajectory and impacting surface) decreased. By decreasing the impact angle, the normal component of the impact velocity decreased. It was shown that lower normal velocity component could cause less DE [28, 29]. It is concluded that thick coating at lower traverse speed led to thick compressed layer of the gas and sharp impact angle between the 
particles' trajectory and surface of the sample, could cause lower DE at lower traverse speed [27].

In CS process, the particles' impact to the substrate can cause bonding or surface activation. Such bonding is observed after an incubation time (induction time) [30, 31]. The incubation time is an important parameter in CS process, which is related to the number of impinging particles and their impact velocity. Succeeding particle impinging on the activated surface is more likely to bond to the surface. At the same spraying condition and powder flow rate, by increasing the traverse speed, the number of impinging particles to the surface area of the substrate decreases, so the incubation time extends. Furthermore, the incubation time is inversely proportional to the deposition flux. Deposition flux refers to the number of deposited particles per unit of time and unit area [32]. On the other hand, by increasing the traverse speed, the substrate's surface temperature decreases, which, in turn, affects the coatings' thickness and incubation time [ r T.The interaction of these phenomena should be considered in terms of the effect of traverse speed on the particles' deposition behavior.

The impact of traverse speed in the very short range on deposition rate in the CS process was reported by some authors [25-27], but the effect of this phenomenon at wide range of traverse speed on DE, coating porosity, surface roughness and microhardness has not been considered yet. The present study aims to achieve the highest DE by alleviating the erosion of the bonded particles. So, the traverse speed is changed to control the deposition flux over the surface area of the substrate. The coatings' properties and microstructural features are investigated by characterization of their cross-section, free surface, and splats. 


\section{Materials and Methods}

\subsection{Numerical simulation}

The momentum and heat transfer from the carrier gas to the particles were simulated to estimate the impact velocity and temperature of the particles using the commercial CFD (Computational Fluid Dynamics) software Fluent 16.2. A 2-D axis-symmetric analysis was performed using a density-based algorithm for a nozzle with an expansion ratio of 5.84 and a divergent length of $127 \mathrm{~mm}$. The effect of turbulence on the nozzle walls was taken into account using the k- $\varepsilon$ turbulence model. Nitrogen was selected as the carrier gas and the particles' density was set to $8330 \mathrm{~kg} / \mathrm{m}^{3}$ with a heat capacity of $430 \mathrm{~J} / \mathrm{Kg}$ per $\mathrm{K}$ (at $300 \mathrm{~K}$ ). The flight of 100 particles, representing the particle size distribution (from the smallest to the largest particle in the feedstock), were simulated independently along the nozzle's central axis for different spraying conditions. By this way, it is possible to understand the effect of spraying conditions on the momentum and heat transfer processes related to every particle size in the distribution. To model the interaction between the propelling gas and the particles, a Lagrangian solution was provided by a Discrete Phase Model available in Fluent [34]; as the particles are simulated individually along the nozzle central axis, the interaction between the particles was not taken into account.

\subsection{Experimental procedures}

The commercially available Stellite 21 powder (ORIC Company Ltd., trade name Stelloric 1388) was used as a feedstock material. Beckman Coulter (LS 13320) laser diffraction particle size analyzer was utilized to study the particle size distribution of the feedstock. The chemical composition of the feedstock is shown in Table 1.

The powder was deposited by high-pressure cold spray equipment (KINETICS $® 4000$ ) on a low-carbon steel $\left(20 * 50 * 5 \mathrm{~mm}^{3}\right)$. The grain size of the substrate was $31.8 \mu \mathrm{m}$ (equal to ASTM 
No. 6.69), which was calculated by the image analysis. The initial microstructure of the substrate consisted of pre-eutectoid ferrite and a small amount of pearlite. The substrate's surface was prepared by grinding it with $\mathrm{SiC}$ sandpaper grit 400 and then cleaning it by acetone.

In the first step, the powder was sprayed based on the simulation results. A $0.75 \mathrm{~mm}$ overlapping between the spray lines was selected to make sure the surface of the substrate was coated thoroughly. Moreover, low traverse speed $\left(20 \mathrm{~mm} \cdot \mathrm{s}^{-1}\right)$ was utilized to achieve dense and thick coatings in single pass deposition and also to inhibit the effect of multi-pass on the coatings' properties $[25,35,36]$. Then the spraying parameters, which lead to the highest DE and microhardness and the lowest porosity, were selected for spraying at different traverse speeds. The free surface of the as-sprayed coatings was evaluated using scanning electron microscopy (SEM). The surface roughness of the as-sprayed coatings was measured by the contact-type roughness measuring instrument.

The deposited samples were cut, mounted, and prepared according to the standard metallography method ASTM E1920. The mounted samples were grinded with 120-1200 grit SiC papers and then polished by 6 and then $1 \mu \mathrm{m}$ monocrystalline diamonds. Nital $5 \%$ and $\mathrm{HCl}+\mathrm{H}_{2} \mathrm{O}_{2}$ solutions were used for etching the microstructure of the substrate and the coating, respectively. The coatings' microstructure and porosity, as well as the bonding quality were characterized by optical microscope and SEM. The porosity was measured in five different zones of the cross-section of each sample and the average value was reported. Matsuzawa micro-hardness tester was used for measuring the coatings' microhardness. The indentation was performed in the middle of the layer with $100 \mathrm{grf}$, and $100 \mu \mathrm{m}$ distance between two points was selected for avoiding errors of the hardness value due to the peripheral region deformation. The average microhardness value of 10-point indentation was reported. 


\section{Results and Discussion}

\subsection{Powder characterization}

The morphology of as-received Stellite 21 powder is shown in Fig. 1a. The feedstock has spherical morphology due to the manufacturing process (atomization process). Fig. 1b shows a featureless structure like a shell around some particles. This structure is acquired due to the high cooling rates of the atomization method [37]. The cross-section of single particles with dendritic microstructure is represented in Fig. 1c. Particle size and flowability (i.e., powder capability in flowing through the powder feeder and injection system) are two essential features of powder in the CS process [38]. Fig. 1d shows a feedstock particle size distribution in which both differential (solid line) and accumulative volume (dashed line) percentages are plotted. The feedstock particles show a Gaussian distribution; where, $d_{\text {mean }}=35 \mu \mathrm{m}, \mathrm{d}_{10}=$ $10 \mu \mathrm{m}$, and $\mathrm{d}_{90}=53 \mu \mathrm{m}$. The flowability of the powder was measured according to the ASTM B213 specifications. Powder flows through Hall powder flowmeter funnel indicate a suitable flowability with a time of $9 \mathrm{sec} / 50 \mathrm{~g}$.

\subsection{Parameters Screening}

The impact velocity and temperature of the particles using box plots are depicted in Fig. 2 where the particles within the distribution are grouped in quartiles for each combination of spraying parameters. Based on the simulation results of the particles' impact temperature and velocity, three different spraying operations were chosen according to Table 2. Spraying was performed at three different conditions: (i) high kinetic, (ii) high thermal and (iii) high kinetic and thermal energy, in order to distinguish the effect of thermal and kinetic energies on the coatings' quality and DE. The measured deposition efficiency of the samples, as well as the coatings' microhardness and thickness are indicated in Table 2. Since the coatings' delamination on the sprayed samples at $40 \mathrm{~mm}$ stand-off distances (samples' ID. 40-800-40, 
40-730-40, and 32-800-40) and in samples 40-730-25 and 32-800-25 leads to the thickness variation, so the thickness and DE values are not reported.

According to the simulation results, by increasing the SoD, the velocity of large particles increases slightly (Fig. 2). Different authors reported the large particle further acceleration after the nozzle exit $[39,40]$. An over-expanded condition of the cold spray nozzle leads to complicated structures of shock wave in it [41]. After the nozzle exit, the interaction between the supersonic jet, the atmosphere and the substrate leads to the formation of shockwave at the nozzle exit and bow shock in front of the substrate, respectively [41-43]. The shockwave after the nozzle exit decrease the gas velocity to subsonic speeds. So, the gas decelerates to lower than the particle velocity and the resultant negative drag force causes the deceleration of particles $[43,44]$. Then the flow is accelerated again to supersonic speeds through this until the next shock recompresses the flow again [42]. There will be a positive drag effect and therefore further acceleration. In the bow shock section, the gas will decelerate abruptly, its velocity will become smaller than the particles velocity and therefore, they will decelerate as well until their impact onto the substrate $[39,40,44-48]$. The impact of the shock wave and bow shock on the particles acceleration or deceleration is related to the particle size and density. Due to the higher mass and corresponding inertia of the large particles rather than the small ones, the influence of negative drag force on the large particles is lower and making them capable of further acceleration at higher standoff distances $[45,46]$.

Due to the low traverse speed, the coatings' thickness is very high $(>1 \mathrm{~mm})$. There is similar effect between the nozzle traverse speed and powder feed rate. High feed rates lead to high localized residual stress between deposits and substrate. High residual stresses can cause the delamination of the deposit [44]. Tan et. al [49] reported the coating delamination at low traverse speed. The residual stress is a function of not only impact condition, but also spraying kinematics and substrate dimension/thermal properties. When the coating and substrate have 
different thermal expansion coefficients, there is an additional effect from the thermal misfit which may greatly influence the final state of stress in the coating/substrate assembly. Thermal misfit strain may change the residual stresses of the system from compressive to tensile [50]. The surface preparation is another parameter which can affect the coating adhesion to the substrate $[22,51]$. In this study, the substrate's surface was prepared by grinding with $\mathrm{SiC}$ sandpaper grit 400 and then cleaning by acetone. Due to the higher value of the critical velocity of such a high strength alloy, adhesion of the coating to the substrate comes from the limited metallurgical bonding site. In addition, the lower temperature of the gas due to the long SoD may lead to less deformability of the previously deposited particles and the substrate and lower mechanical interlocking of the coatings to the substrate. These phenomena can lead to the coating delamination at higher SoDs. The cracks and delamination are considered as the sign of the poor quality of the coatings. The properties of good quality samples are further investigated in terms of the particles' flattening ratio and porosity.

The cross-sections of the sprayed samples at $10 \mathrm{~mm}$ SoD are represented in Fig. 3. In these micrographs, the substrates are etched to distinguish the interface and the entrapped particle on the substrate. Due to the higher corrosion resistance of Stellite 21 compared to steel substrate, the etching agent (Nital 5\%) did not reveal the coating's microstructure. The coatings microstructure was revealed by $\mathrm{HCl}-\mathrm{H}_{2} \mathrm{O}_{2}$ solution. The etched microstructure of the coatings is represented in Fig. $3 \mathrm{~d}$ to $\mathrm{f}$. The measured values of the particles' flattening ratio and the amount of porosity are shown in Fig. 4. Almost the same value of the impact temperature of the particles in samples 40-800-10 and 32-800-10 (according to the simulation results) as well as their higher impact velocity in sample 40-800-10 led to the better adhesion of the coating to the substrate and less porosity. At the nearly same particle and substrate temperature (due to the same gas temperature in samples 40-800-10 and 32-800-10), the particles' physical entrapment in the substrate surface at higher impact velocity caused better mechanical 
locking, which can be suggested as a reason for the better bonding to the substrate [52]. The entrapped particles are represented by black arrows in Fig. 3a for sample 40-800-10. The average values of the flattening ratio of 30 particles were calculated at the etched cross-section of the coatings and the results are reported in Fig. 4. In sample 40-80-10, the highest flattening ratio and lowest porosity are observed. These may be attributed to the highest impact velocity and impact temperature of the particles in the sample [53]. A higher flattening ratio means a higher plastic deformation of the particles and the higher compressive residual stress in the coating. In Fig. 5, the bonding between the coating and the substrate and the cohesive strength of the coatings were evaluated by SEM micrograph and Vickers' indentation test, respectively. The coating cross-sections were compared in terms of presence of cracks and delamination which means the lower bonding strength of the coatings. As represented in Figs. $3 \mathrm{~b}$ and $5 \mathrm{~b}$, there are cracks at the interface of the coating 32-800-10. Also, the higher value of porosity was observed in the coating 40-730-10 (Figs. $3 c$ and 5c) rather than 40-800-10. The presence of porosity and interface cracks in the coating can cause the lower bonding. The Vickers' indentation points are shown in Fig. 5d-f. According to these images, cracks growth on the corner of indentation points is considered as a criterion for the lower cohesion strength. In the sample with the highest kinetic and thermal energy (40-800-10), the particles experience more deformation. Furthermore, the inter-particle crack propagation is hindered by the higher mechanical interlocking and compressive residual stress in the coating $[25,54]$. On the other side, the cohesion strength of sample 32-800-10 is lower than those of 40-800-10 and 40-73010 and large cracks are visible around the indentation point. The lower flattening ratio of the particles in sample 32-800-10 may cause the lower cohesive strength. In order to consider the effect of spraying parameters on the deformation and deposition behavior of single particles, the wipe test was performed for sample 40-800-10, which had the lowest porosity, highest 
microhardness, a better bonding between the particles and also between the coating and the substrate.

\subsection{Particles splats}

In the wipe test, using low feeding rate and high traverse speed (here, $1000 \mathrm{~mm} \cdot \mathrm{s}^{-1}$ has been used), a limited number of particles are sprayed and the bonding and rebounding events of a single particle are investigated [55]. Rebounding occurs when the particle does not have enough total energy (thermal plus kinetic energy) to bonding to the substrate. The surface morphology of the wipe test sample is shown in Fig. 6. For a better understanding of the particles' deformation behavior, the surface of the sample was investigated in the top and $70^{\circ}$ tilt view by SEM (Figs. 6d-f). Despite the lower deformability of cobalt-based hightemperature alloys, the wipe test showed that in the selected parameters, most of the particles were deformed and bonded to the substrate. The metal jets around the bonded particles are represented by black arrow in Figs. $6 \mathrm{~b}$ and $6 \mathrm{c}$, which implies the bonding occurred between the particles and substrate [56]. There are just a few craters on the substrate (Fig. 6a), which come from the big particles' rebounding [30, 57]. It means that some large particles do not reach the critical velocity. It is observed that the medium size particles are deformed and bonded to the substrate. Additionally, there are some small particles that are not deformed and just plunged to the substrate. Moreover, there is no sign of jet around them. Substrate deformation around the small-embedded particles prevents their rebounding. This observation approves the findings of other authors about the increase of critical velocity by decreasing the particle size $[46,58]$. Overall, the wipe test shows the potential for high deposition efficiency at the first layer deposition. 


\subsection{Influence of traverse speed}

The main differences between the wipe test and the initial screening tests are the deposition flux over the surface area of the substrate and deposit layer thickness. In the wipe test, the probability of collision between the bonded and following particles decreases. Besides, the wipe test is performed in one line, while in the initial screening tests, the step size of $0.75 \mathrm{~mm}$ is selected between the successive lines, which leads to the thick coating. Therefore, it seems that the deposition flux, higher thickness of the coatings and step size are the sources of low DE at low traverse speed. At lower traverse speed the thickness of deposited layer increased and the impact angle of the particles decreased and the normal component of the impact velocity decreased [27]. It was shown that lower normal velocity component could cause less DE $[28,29]$. Then, new tests are performed to identify the effect of coating thickness, deposition flux and step size on the DE. The influence of the coatings' thickness was investigated by deposition of single tracks at different travers speeds. There are two main techniques for controlling the deposition flux over the surface area of substrate i) decreasing the feeding rate, and ii) increasing the traverse speed of the nozzle. The latter technique is used because controlling the traverse speed of the nozzle is more facile than adjusting the feeding rate. The flux of particles per unit area of the substrate alters by changing the nozzle's traverse speed. So, different traverse speeds from 20 to $400 \mathrm{~mm} \cdot \mathrm{s}^{-1}$ were selected for performing the new tests. Finally, the $3 \mathrm{~mm}$ step size is used for investigating the effect of step size on DE.

Initially, the powder was sprayed in single track at different traverse speed (20 to $\left.400 \mathrm{~mm} \cdot \mathrm{s}^{-1}\right)$ to investigate the effect of the layer thickness on deposition behavior. Optical micrographs of the resultant tracks' cross-section are represented in Fig. 7. As shown in these optical micrographs, at $20 \mathrm{~mm} \cdot \mathrm{s}^{-1}$ traverse speed, the thickness of the deposited track was about $250 \mu \mathrm{m}$. The impact angle deviation from normal direction is less than $6^{\circ}$. So, the effect of track 
thickness on the normal component of the particles' impact velocity, the particles' deposition behavior and resultant coating properties is negligible [29, 39].

To calculate the deposition flux, the total flow rate of the particles from the powder feeder (0.67 gr.s $\left.{ }^{-1}\right)$ was delineated. The flux of the particle per unit area of the substrate was estimated by $\mathrm{P}_{\mathrm{f}}=\mathrm{F}_{\mathrm{r}} / \mathrm{L}_{\mathrm{n}} . \mathrm{D}_{\mathrm{e}}$; where, $\mathrm{F}_{\mathrm{r}}, \mathrm{L}_{\mathrm{n}}$, and $\mathrm{D}_{\mathrm{e}}$ are the particle flow rate $\left(\mathrm{gr} \cdot \mathrm{s}^{-1}\right)$, the length of the nozzle displacement per second $(\mathrm{mm})$, and the nozzle's exit diameter $(\mathrm{mm})$, respectively. Due to the various sizes of the particles of feedstock, their interaction with the gas flow is different. So, the particles' distribution over the surface is not uniform. The effect of particle size on their distribution over the substrate has been investigated by some authors using the simulation and experimental results $[15,59]$. To simplify the analyses, we assumed that the particles are uniformly distributed over the exposed area of the substrate. It was further assumed that the area of the exposed surface coincides with the area of the jet crosssection and also with the nozzle's exit area [60]. The calculated values of the deposition flux at different traverse speeds are shown in Fig. 8.

By increasing the traverse speed from 20 to $400 \mathrm{~mm} \cdot \mathrm{s}^{-1}$, the coating thickness declines due to the less flux of the particles over the substrate at higher traverse speeds. SEM micrographs of the coatings' cross-sections are shown in Fig. 9. In these micrographs the substrates are etched to distinguish the interface of the coatings. Interestingly, spraying at different traverse speeds led to different DE values. Variations of DE by traverse speed are plotted in Fig. 10. As illustrated, the DE increases very fast at traverse speed values ranging from 20 to $100 \mathrm{~mm} \cdot \mathrm{s}^{-1}$. Then the slope of the plot decreases at traverse speed values ranging from 100 to $300 \mathrm{~mm} \cdot \mathrm{s}^{-1}$. Finally, a down-trend of the DE is observed. Also the surface roughness and the thickness of the coatings decline by an increase in the traverse speed. In order to distinguish the effect of traverse speed, the surface morphology of the sprayed sample was examined by SEM. 
The schematic view of the particles' impact on the substrate at 20,100 , and $400 \mathrm{~mm} \cdot \mathrm{s}^{-1}$ traverse speeds and the SEM micrographs of the coatings are represented in Fig. 11. At lower traverse speeds, more sign of erosion or crater is observed at the surface of the coating (white arrows in the SEM micrographs). The erosion of the deposited particle occurs due to the subsequent collision of the particles. A larger number of craters and flat area (represented by red closed line) suggests that more particles have been detached due to the erosion. As the traverse speed increases, the erosion level decreases and the DE increases. Hence, it seems that the erosion of the particles can be the reason of the lower DE at lower traverse speed. The particle distribution in the nozzle is related to the nozzle cross-section and evenly distributed coating achieved by the elliptical cross-section [61] rather than spherical and square nozzles. Samarech et al. [62] found that smaller particles were more affected by gas flow and were more deviated due to their Stokes numbers. At lower traverse speeds, there is a higher concentration of the samesize particles in the small area of the substrate. By increasing the traverse speed, the nonuniformity of the particles' distribution decreases which can affect the surface roughness. At higher traverse speeds the deposition flux over the surface area decreases. The less intensive deposition flux over the surface of the substrate leads to the less probability of particles collision to the bonded particles (Figs. 11a-c). This phenomenon reduces the hammering effect and thus leads to higher porosity and lower microhardness of the coating. Klinkov et al. [31] showed that induction time is inversely proportional to the deposition flux. So, the surface preparation is not performed very well; then the bonding of first incident particles is affected by insufficient surface preparation. Additionally, at higher traverse speeds, the substrate's surface temperature decreases; this in turn, affects the coatings' thickness and bonding strength $[\pi, \leqslant 9, \pi]$. All these can affect the deformation and deposition behavior of particles. Furthermore, the lower DE at $400 \mathrm{~mm} \cdot \mathrm{s}^{-1}$ may come from insufficient surface preparation and lower temperature of the substrate. It is found that the porosity of the coating increases 
gradually with the increment of nozzle transverse speed. The higher value of porosity may come from the lower particles plastic deformation at higher traverse speed. In Fig. 11f, the existence of porosity at the surface of the as-sprayed sample (black arrows) confirms the less peening of the bonded particles at this sample. Also there is no flat area which comes from the erosion of bonded particles. The microhardness and porosity of the coatings at different traverse speeds are represented in Fig. 12. The measurement of the coating microhardness for $400 \mathrm{~mm} \cdot \mathrm{s}^{-1}$ traverse speed has not been performed due to the lower thickness and higher porosity of the coatings. The coating with highest achievable DE along with lower porosity is more favorable. Accordingly, the traverse speed of $100 \mathrm{~mm} \cdot \mathrm{s}^{-1}$ was selected as the proper traverse speed.

The final test was done for finding the effect of step size on the coatings' deposition efficiency. By increasing the step size from 0.75 to $3 \mathrm{~mm}$, the measured value of DE was almost the same. In other words, the effect of step size on the DE at $100 \mathrm{~mm} \cdot \mathrm{s}^{-1}$ traverse speed was negligible.

\section{Conclusions}

In this study, the effect of the nozzle's traverse speed (20 to $\left.400 \mathrm{~mm} \cdot \mathrm{s}^{-1}\right)$ on the cold spraying of Stellite (St21) powder was investigated. The porosity, coating microhardness, and DE were deliberated as the criteria for the process optimization. Also the erosion evidence was shown and the erosion mitigation strategy was proposed in order to increase deposition efficiency. Overall, the conclusions of this study can be outlined as follows:

1. Although Stellite 21 powders have a high strength, the deformation and deposition behavior of single particles showed that most of the particles are deformed and bonded to the substrate. The jet ring around the particle is visible for most of the particles, meaning that bonding has occurred at these parameters. 
2. Spraying at different traverse speeds $\left(20-400 \mathrm{~mm} \cdot \mathrm{s}^{-1}\right)$ led to the different values of DE. The DE increased from $23 \%$ at $20 \mathrm{~mm} \cdot \mathrm{s}^{-1}$ to $48 \%$ at $300 \mathrm{~mm} \cdot \mathrm{s}^{-1}$ and then decreased to $30 \%$ at $400 \mathrm{~mm} \cdot \mathrm{s}^{-1}$.

3. By increasing the traverse speed from 20 to $400 \mathrm{~mm} / \mathrm{s}$, the coatings' porosity increased from $0.82 \%$ to $9.4 \%$ and their microhardness decreased from 612 to $474 \mathrm{HV}$. These variations can be due to the hammering effect of incident particles and the substrate's surface temperature. Furthermore, at traverse speeds higher than $100 \mathrm{~mm} \cdot \mathrm{s}^{-1}$, the coatings' quality was decreased by increasing the porosity and decreasing the thickness and microhardness.

4. DE curve exhibited three different zones. By increasing the traverse speed from 20 to $100 \mathrm{~mm} \cdot \mathrm{s}^{-1}$, the DE increased sharply. Then the slope of the curve dropped at the traverse speed values ranging from 100 to $300 \mathrm{~mm} . \mathrm{s}^{-1}$. The decrease of the DE at $400 \mathrm{~mm} \cdot \mathrm{s}^{-1}$ traverse speed can be attributed to the lower temperature of the surface and insufficient surface preparation before deposition. 


\section{References}

[1] N. Cinca, J.M. Guilemany, Structural and properties characterization of stellite coatings obtained by cold gas spraying, Surface and Coatings Technology, 220 (2013) 90-97. https://doi.org/10.1016/j.surfcoat.2012.11.026.

[2] S. Zhang, Aerospace Materials Handbook, in, Taylor \& Francis, Boca Raton, 2013.

[3] J.R. Davis, Nickel, cobalt, and their alloys, ASM international, 2000.

[4] D.H.E. Persson, On the Mechanisms behind the Tribological Performance of Stellites, in: Digital Comprehensive Summaries of Uppsala Dissertations from the Faculty of Science and Technology, Acta Universitatis Upsaliensis, Uppsala, 2005, pp. 51.

[5] G.P. Rajeev, M. Kamaraj, S.R. Bakshi, Hardfacing of AISI H13 tool steel with Stellite 21 alloy using cold metal transfer welding process, Surface and Coatings Technology, 326 (2017) 63-71. https://doi.org/10.1016/j.surfcoat.2017.07.050.

[6] S. Apay, B. Gulenc, Wear properties of AISI 1015 steel coated with Stellite 6 by microlaser welding, Materials \& Design, 55 (2014) 1-8. http://dx.doi.org/10.1016/j.matdes.2013.09.056.

[7] A. Kusmoko, D. Dunne, H. Li, D. Nolan, Effect of Two Different Energy Inputs for Laser Cladding of Stellite 6 on P91 and P22 Steel Substrates, Procedia Materials Science, 6 (2014) 26-36. http://dx.doi.org/10.1016/j.mspro.2014.07.005.

[8] R. Singh, D. Kumar, S.K. Mishra, S.K. Tiwari, Laser cladding of Stellite 6 on stainless steel to enhance solid particle erosion and cavitation resistance, Surface and Coatings Technology, 251 (2014) 87-97. http://dx.doi.org/10.1016/j.surfcoat.2014.04.008.

[9] B. Li, Y. Jin, J. Yao, Z. Li, Q. Zhang, Solid-state fabrication of WCp-reinforced Stellite-6 composite coatings with supersonic laser deposition, Surface and Coatings Technology, 321 (2017) 386-396. https://doi.org/10.1016/j.surfcoat.2017.04.062.

[10] F. Luo, R. Lupoi, A. Cockburn, M. Sparkes, W. O'Neill, J.-h. Yao, Characteristics of Stellite 6 Deposited by Supersonic Laser Deposition Under Optimized Parameters, Journal of Iron and Steel Research, International, 20 (2013) 52-57. http://dx.doi.org/10.1016/S1006-706X(13)60056-4.

[11] H. Assadi, H. Kreye, F. Gärtner, T. Klassen, Cold spraying - A materials perspective, Acta Materialia, (2016) 382-407. https://doi.org/10.1016/j.actamat.2016.06.034.

[12] R. Singh, S. Schruefer, S. Wilson, J. Gibmeier, R. Vassen, Influence of coating thickness on residual stress and adhesion-strength of cold-sprayed Inconel 718 coatings, Surface and Coatings Technology, 350 (2018) 64-73. https://doi.org/10.1016/j.surfcoat.2018.06.080.

[13] A. Moridi, S.M. Hassani-Gangaraj, M. Guagliano, M. Dao, Cold spray coating: Review of material systems and future perspectives, Surface Engineering, 30 (2014) 369-395. https://doi.org/10.1179/1743294414Y.0000000270.

[14] Z.B. Zhao, B.A. Gillispie, J.R. Smith, Coating deposition by the kinetic spray process, Surface and Coatings Technology, 200 (2006) 4746-4754. https://doi.org/10.1016/j.surfcoat.2005.04.033.

[15] M. Faizan-Ur-Rab, S.H. Zahiri, P.C. King, C. Busch, S.H. Masood, M. Jahedi, R. Nagarajah, S. Gulizia, Utilization of Titanium Particle Impact Location to Validate a 3D Multicomponent Model for Cold Spray Additive Manufacturing, Journal of Thermal Spray Technology, 26 (2017) 1874-1887. https://doi.org/10.1007/s11666-017-0628-4.

[16] V.K. Champagne, The cold spray materials deposition process: fundamentals and applications, Elsevier, 2007.

[17] R. Maestracci, A. Sova, M. Jeandin, J.M. Malhaire, I. Movchan, P. Bertrand, I. Smurov, Deposition of composite coatings by cold spray using stainless steel 316L, copper and Tribaloy T-700 powder $\begin{array}{llllll}\text { mixtures, } & \text { Surface and } & \text { Coatings Technology, } 287 & \text { (2016) }\end{array}$ https://doi.org/10.1016/i.surfcoat.2015.12.065.

[18] M. Villa, S. Dosta, J.M. Guilemany, Optimization of 316L stainless steel coatings on light alloys using Cold Gas Spray, Surface and Coatings Technology, 235 (2013) 220-225. https://doi.org/10.1016/i.surfcoat.2013.07.036. 
[19] M.R. Rokni, S.R. Nutt, C.A. Widener, V.K. Champagne, R.H. Hrabe, Review of Relationship Between Particle Deformation, Coating Microstructure, and Properties in High-Pressure Cold Spray, Journal of Thermal Spray Technology, (2017) 1308-1355. https://doi.org/10.1007/s11666-017-0575-0.

[20] M.P.d.M. Couto, Cold Spray Deposition of WC-Co Cermets, in: thermal spray center, Barcelona, Barcelona, 2014, pp. 148.

[21] R. Fernandez, B. Jodoin, Effect of Particle Morphology on Cold Spray Deposition of Chromium Carbide-Nickel Chromium Cermet Powders, Journal of Thermal Spray Technology, (2017). http://dx.doi.org/10.1007/s11666-017-0580-3.

[22] A. Nastic, M. Vijay, A. Tieu, S. Rahmati, B. Jodoin, Experimental and Numerical Study of the Influence of Substrate Surface Preparation on Adhesion Mechanisms of Aluminum Cold Spray Coatings on 300M Steel Substrates, Journal of Thermal Spray Technology, (2017). https://doi.org/10.1007/s11666-017-0602-1.

[23] N. Cinca, J. Guilemany, Cold Gas Sprayed Stellite-6 Coatings and their Wear Resistance, J Material Sci Eng, 2 (2013) 2169-0022.1000. https://doi.org/10.4172/2169-0022.1000122.

[24] N. Cinca, E. López, S. Dosta, J.M. Guilemany, Study of stellite-6 deposition by cold gas spraying, $\begin{array}{lllll}\text { Surface and } \quad \text { Coatings } & \text { Technology, } & & \end{array}$ http://dx.doi.org/10.1016/j.surfcoat.2013.06.120.

[25] P. Richer, A. Zúñiga, M. Yandouzi, B. Jodoin, CoNiCrAlY microstructural changes induced during Cold Gas Dynamic Spraying, Surface and Coatings Technology, 203 (2008) 364-371. https://doi.org/10.1016/j.surfcoat.2008.09.014.

[26] V. Varadaraajan, P. Mohanty, Design and optimization of rectangular cold spray nozzle: Radial injection angle, expansion ratio and traverse speed, Surface and Coatings Technology, 316 (2017) 246254. https://doi.org/10.1016/j.surfcoat.2017.03.005.

[27] D. Kotoban, S. Grigoriev, A. Okunkova, A. Sova, Influence of a shape of single track on deposition efficiency of 316L stainless steel powder in cold spray, Surface and Coatings Technology, (2016). https://doi.org/10.1016/i.surfcoat.2016.10.052.

[28] S. Yin, X.F. Wang, W.Y. Li, B.P. Xu, Numerical study on the effect of substrate angle on particle impact velocity and normal velocity component in cold gas dynamic spraying based on CFD, Journal of Thermal Spray Technology, 19 (2010) 1155-1162. https://doi.org/10.1007/s11666-010-9510-3.

[29] K. Binder, J. Gottschalk, M. Kollenda, F. Gärtner, T. Klassen, Influence of Impact Angle and Gas Temperature on Mechanical Properties of Titanium Cold Spray Deposits, Journal of Thermal Spray Technology, 20 (2011) 234-242. https://doi.org/10.1007/s11666-010-9557-1.

[30] S.V. Klinkov, V.F. Kosarev, Measurements of cold spray deposition efficiency, Journal of Thermal Spray Technology, 15 (2006) 364-371. https://doi.org/10.1361/105996306X124365.

[31] S.V. Klinkov, V.F. Kosarev, M. Rein, Cold spray deposition: Significance of particle impact phenomena, Aerospace Science and Technology, 9 (2005) 582-591. https://doi.org/10.1016/j.ast.2005.03.005.

[32] K. Higashitani, H. Masuda, H. Yoshida, Powder technology: fundamentals of particles, powder beds, and particle generation, CRC Press, 2006.

[33] C. Chen, Y. Xie, C. Verdy, R. Huang, H. Liao, Z. Ren, S. Deng, Numerical investigation of transient coating build-up and heat transfer in cold spray, Surface and Coatings Technology, 326 (2017) 355365. http://dx.doi.org/10.1016/j.surfcoat.2017.07.069.

[34] Fluent 6.2 User Guide Manual, in.

[35] A. Moridi, S.M.H. Gangaraj, S. Vezzu, M. Guagliano, Number of Passes and Thickness Effect on Mechanical Characteristics of Cold Spray Coating, Procedia Engineering, 74 (2014) 449-459. https://doi.org/10.1016/i.proeng.2014.06.296.

[36] S.A. Alidokht, P. Manimunda, P. Vo, S. Yue, R.R. Chromik, Cold spray deposition of a Ni-WC composite coating and its dry sliding wear behavior, Surface and Coatings Technology, 308 (2016) 424434. https://doi.org/10.1016/i.surfcoat.2016.09.089. 
[37] F. Duflos, J.-F. Stohr, Comparison of the quench rates attained in gas-atomized powders and meltspun ribbons of Co- and $\mathrm{Ni}$-base superalloys: influence on resulting microstructures, Journal of Materials Science, 17 (1982) 3641-3652. https://doi.org/10.1007/bf00752209.

[38] J. Villafuerte, W. Birtch, J. Wang, Commercial cold spray equipment and automation, in: Modern Cold Spray: Materials, Process, and Applications, Springer International Publishing, 2015, pp. 253-273. [39] D.L. Gilmore, R.C. Dykhuizen, R.A. Neiser, T.J. Roemer, M.F. Smith, Particle velocity and deposition efficiency in the cold spray process, Journal of Thermal Spray Technology, 8 (1999) 576-582. https://doi.org/10.1361/105996399770350278.

[40] S.H. Zahiri, W. Yang, M. Jahedi, Characterization of cold spray titanium supersonic jet, Journal of Thermal Spray Technology, 18 (2009) 110-117. 10.1007/s11666-008-9278-x.

[41] H. Katanoda, T. Matsuoka, K. Matsuo, Experimental study on shock wave structures in constantarea passage of cold spray nozzle, Journal of Thermal Science, 16 (2007) 40-45. https://doi.org/10.1007/s11630-007-0040-3.

[42] F. Gnani, H. Zare-Behtash, K. Kontis, Pseudo-shock waves and their interactions in high-speed intakes, Progress in Aerospace Sciences, 82 (2016) 36-56. https://doi.org/10.1016/i.paerosci.2016.02.001.

[43] M. Winnicki, T. Piwowarczyk, A. Malachowska, General description of cold sprayed coatings formation and of their properties, Bulletin of the Polish Academy of Sciences: Technical Sciences, (2018).

[44] S. Yin, P. Cavaliere, B. Aldwell, R. Jenkins, H. Liao, W. Li, R. Lupoi, Cold spray additive manufacturing and repair: Fundamentals and applications, Addit. Manuf., 21 (2018) 628-650. https://doi.org/10.1016/i.addma.2018.04.017.

[45] M. Faizan-Ur-Rab, S.H. Zahiri, S.H. Masood, T.D. Phan, M. Jahedi, R. Nagarajah, Application of a holistic 3D model to estimate state of cold spray titanium particles, Materials \& Design, 89 (2016) 1227-1241. https://doi.org/10.1016/i.matdes.2015.10.075.

[46] H. Assadi, T. Schmidt, H. Richter, J.O. Kliemann, K. Binder, F. Gärtner, T. Klassen, H. Kreye, On parameter selection in cold spraying, Journal of Thermal Spray Technology, 20 (2011) 1161-1176. https://doi.org/10.1007/s11666-011-9662-9.

[47] W.Y. Li, C. Zhang, X.P. Guo, G. Zhang, H.L. Liao, C.J. Li, C. Coddet, Effect of standoff distance on coating deposition characteristics in cold spraying, Materials \& Design, 29 (2008) 297-304. https://doi.org/10.1016/j.matdes.2007.02.005.

[48] J. Pattison, S. Celotto, A. Khan, W. O'Neill, Standoff distance and bow shock phenomena in the Cold Spray process, Surface and Coatings Technology, 202 (2008) 1443-1454. https://doi.org/10.1016/j.surfcoat.2007.06.065.

[49] A.W.-Y. Tan, W. Sun, Y.P. Phang, M. Dai, I. Marinescu, Z. Dong, E. Liu, Effects of Traverse Scanning Speed of Spray Nozzle on the Microstructure and Mechanical Properties of Cold-Sprayed Ti6Al4V Coatings, Journal of Thermal Spray Technology, (2017). https://doi.org/10.1007/s11666-017-0619-5.

[50] Z. Arabgol, H. Assadi, T. Schmidt, F. Gärtner, T. Klassen, Analysis of thermal history and residual stress in cold-sprayed coatings, Journal of Thermal Spray Technology, 23 (2014) 84-90. https://doi.org/10.1007/s11666-013-9976-x.

[51] M.M. Sharma, T.J. Eden, B.T. Golesich, Effect of Surface Preparation on the Microstructure, Adhesion, and Tensile Properties of Cold-Sprayed Aluminum Coatings on AA2024 Substrates, Journal of Thermal Spray Technology, 24 (2014) 410-422. https://doi.org/10.1007/s11666-014-0175-1.

[52] F. Meng, D. Hu, Y. Gao, S. Yue, J. Song, Cold-spray bonding mechanisms and deposition efficiency prediction for particle/substrate with distinct deformability, Materials and Design, 109 (2016) 503510. https://doi.org/10.1016/i.matdes.2016.07.103.

[53] S.H. Zahiri, D. Fraser, S. Gulizia, M. Jahedi, Effect of processing conditions on porosity formation in cold gas dynamic spraying of copper, Journal of Thermal Spray Technology, 15 (2006) 422-430. https://doi.org/10.1361/105996306X124437. 
[54] V. Luzin, K. Spencer, M.X. Zhang, Residual stress and thermo-mechanical properties of cold spray $\begin{array}{lllll}\text { metal } \quad \text { coatings, } & \text { 1259-1270. }\end{array}$ https://doi.org/10.1016/i.actamat.2010.10.058.

[55] H. Assadi, F. Gärtner, T. Stoltenhoff, H. Kreye, Bonding mechanism in cold gas spraying, Acta Materialia, 51 (2003) 4379-4394. https://doi.org/10.1016/S1359-6454(03)00274-X.

[56] C. Chen, Y. Xie, S. Yin, M.P. Planche, S. Deng, R. Lupoi, H. Liao, Evaluation of the interfacial bonding between particles and substrate in angular cold spray, Materials Letters, 173 (2016) 76-79. https://doi.org/10.1016/i.matlet.2016.03.036.

[57] N. Cinca, M. Barbosa, S. Dosta, J.M. Guilemany, Study of Ti deposition onto Al alloy by cold gas spraying, Surface and Coatings Technology, 205 (2010) 1096-1102. https://doi.org/10.1016/i.surfcoat.2010.03.061.

[58] T. Schmidt, F. Gärtner, H. Assadi, H. Kreye, Development of a generalized parameter window for cold spray deposition, Acta Materialia, 54 (2006) 729-742. https://doi.org/10.1016/i.actamat.2005.10.005.

[59] S. Yin, M. Meyer, W. Li, H. Liao, R. Lupoi, Gas Flow, Particle Acceleration, and Heat Transfer in Cold Spray: A review, Journal of Thermal Spray Technology, (2016) 1-23. https://doi.org/10.1007/s11666016-0406-8.

[60] A. Papyrin, V. Kosarev, S. Klinkov, A. Alkimov, V. Fomin, Chapter 2 - High-velocity interaction of particles with the substrate. Experiment and modeling, in: Cold Spray Technology, Elsevier, Oxford, 2007, pp. 33-118.

[61] H. Tabbara, S. Gu, D.G. McCartney, T.S. Price, P.H. Shipway, Study on process optimization of cold gas spraying, Journal of Thermal Spray Technology, 20 (2011) 608-620. https://doi.org/10.1007/s11666-010-9564-2.

[62] B. Samareh, A. Dolatabadi, A three-dimensional analysis of the cold spray process: The effects of substrate location and shape, Journal of Thermal Spray Technology, 16 (2007) 634-642. https://doi.org/10.1007/s11666-007-9082-z.

[63] S. Yin, X. Suo, Y. Xie, W. Li, R. Lupoi, H. Liao, Effect of substrate temperature on interfacial bonding for cold spray of $\mathrm{Ni}$ onto $\mathrm{Cu}$, Journal of Materials Science, 50 (2015) 7448-7457. https://doi.org/10.1007/s10853-015-9304-6. 
Tables

Table 1. The chemical composition of the feedstock, Stellite 21 in wt.\%

\begin{tabular}{cccccccc}
\hline Co & C & Si & Cr & Ni & Mo & W & Fe \\
\hline bal. & 0.3 & 1 & 28 & 2.5 & 5.5 & $>0.5$ & $>2$ \\
\hline
\end{tabular}


Table 2. The samples nomination, process parameters, and the coating deposition efficiency (DE) and surface quality

\begin{tabular}{|c|c|c|c|c|c|c|c|}
\hline Sample ID & $\begin{array}{c}\text { Gas } \\
\text { Pressure } \\
\text { (bar) } \\
\end{array}$ & $\begin{array}{c}\text { Gas } \\
\text { Temperature } \\
\left({ }^{\circ} \mathrm{C}\right) \\
\end{array}$ & $\begin{array}{c}\text { SoD } \\
(\mathbf{m m})\end{array}$ & $\begin{array}{l}\text { DE } \\
(\%)\end{array}$ & $\begin{array}{l}\text { Micro-hardness } \\
\text { (HV) }\end{array}$ & $\begin{array}{l}\text { Thickness } \\
\quad(\mu \mathrm{m})\end{array}$ & Coating Condition \\
\hline $40-800-10$ & \multirow{3}{*}{40} & \multirow{3}{*}{800} & 10 & 23 & $656 \pm 34$ & $1357 \pm 14$ & Good Quality \\
\hline $40-800-25$ & & & 25 & 22 & $616 \pm 57$ & \multirow[t]{2}{*}{$1280 \pm 25$} & Traverse Crack \\
\hline $40-800-40$ & & & 40 & - & $555 \pm 32$ & & Partial Delamination \\
\hline $32-800-10$ & \multirow{3}{*}{32} & \multirow{3}{*}{800} & 10 & 22 & $565 \pm 45$ & $1364 \pm 16$ & Good Quality \\
\hline $32-800-25$ & & & 25 & - & $593 \pm 37$ & \multirow[t]{2}{*}{-} & Crack \& Delamination \\
\hline $32-800-40$ & & & 40 & - & $577 \pm 61$ & & Partial Delamination \\
\hline $40-730-10$ & \multirow{3}{*}{40} & \multirow{3}{*}{730} & 10 & 21 & $643 \pm 18.5$ & $1215 \pm 18$ & Good Quality \\
\hline $40-730-25$ & & & 25 & - & $622 \pm 53$ & \multirow[t]{2}{*}{-} & Crack \& Delamination \\
\hline $40-730-40$ & & & 40 & - & $535 \pm 97$ & & Partial Delamination \\
\hline
\end{tabular}




\section{List of figure captions}

Fig. 1. SEM micrographs of a) initial powder morphology, b) ultrafine microstructure shell around the particle surface, c) dendritic microstructure of feedstock, and d) particle size distribution (solid line) and accumulative volume percentages (dashed line) obtained from the LS test.

Fig. 2. Impact velocity and temperature of the particles at different gas pressure $(\mathrm{P})$ and temperature (T) values for 10,25 , and $40 \mathrm{~mm}$ SoDs.

Fig. 3. Optical micrographs of the coating's cross-section at different pressure and temperatures at $10 \mathrm{~mm}$ stand-off distance: a) $40-800$, b) $32-800$, c) $40-730$, the samples were etched with Nital $5 \%$ to differentiate the interface of the coatings. The optical micrograph of the coatings etched with $\mathrm{HCl}+\mathrm{H} 2 \mathrm{O} 2$ solution d) 40-800, e) 32-800 and f) 40-730 samples.

Fig. 4. Effect of gas pressure and temperature on the particles' flattening ratio and porosity (\%) at $10 \mathrm{~mm}$ SoD.

Fig. 5. The SEM micrographs of the coatings' interface in a) 40-800, b) 32-800, and c) 40-730 samples represent the poor bonding of the particles in sample 32-800. The indentation point in samples d) 40-800, e) 32-800, and f) 40-730, and the cracks propagation in sample 32-800 are observed around the indentation point's edge.

Fig. 6. SEM micrographs of the surface of the wipe tested samples: (a-c) the top view. The black arrows represent the metal jet around the single particles (The craters are shown by white arrows), and (d-e) $70^{\circ}$ tilt view.

Fig. 7. Optical micrograph of the single layer deposited at $40 \mathrm{bar}, 800^{\circ} \mathrm{C}$ and $10 \mathrm{~mm} \mathrm{SoD}$ at different traverse speeds ranging from 20 to $400 \mathrm{~mm} \cdot \mathrm{s}^{-1}$.

Fig. 8. Variation of deposition flux with traverse speed.

Fig. 9. SEM micrographs of the coatings' cross-section at different traverse speeds $\left(\mathrm{mm} . \mathrm{s}^{-1}\right)$. The substrate was etched with Nital 5\% for better distinguishing the coating interface.

Fig. 10. Effect of traverse speed on the coatings' DE, thickness, and surface roughness.

Fig. 11. Effect of traverse speed on the coating formation represented schematically for the samples sprayed at a) 20, b) 100, and c) $400 \mathrm{~mm} \cdot \mathrm{s}^{-1}$; SEM micrographs of the surface of the as-sprayed coatings (c-d). Black and white arrows in the SEM micrographs show the porosities and erosion craters, respectively.

Fig. 12. Variations of the coatings' micro-hardness and porosity at different traverse speeds. 


\section{Figures}
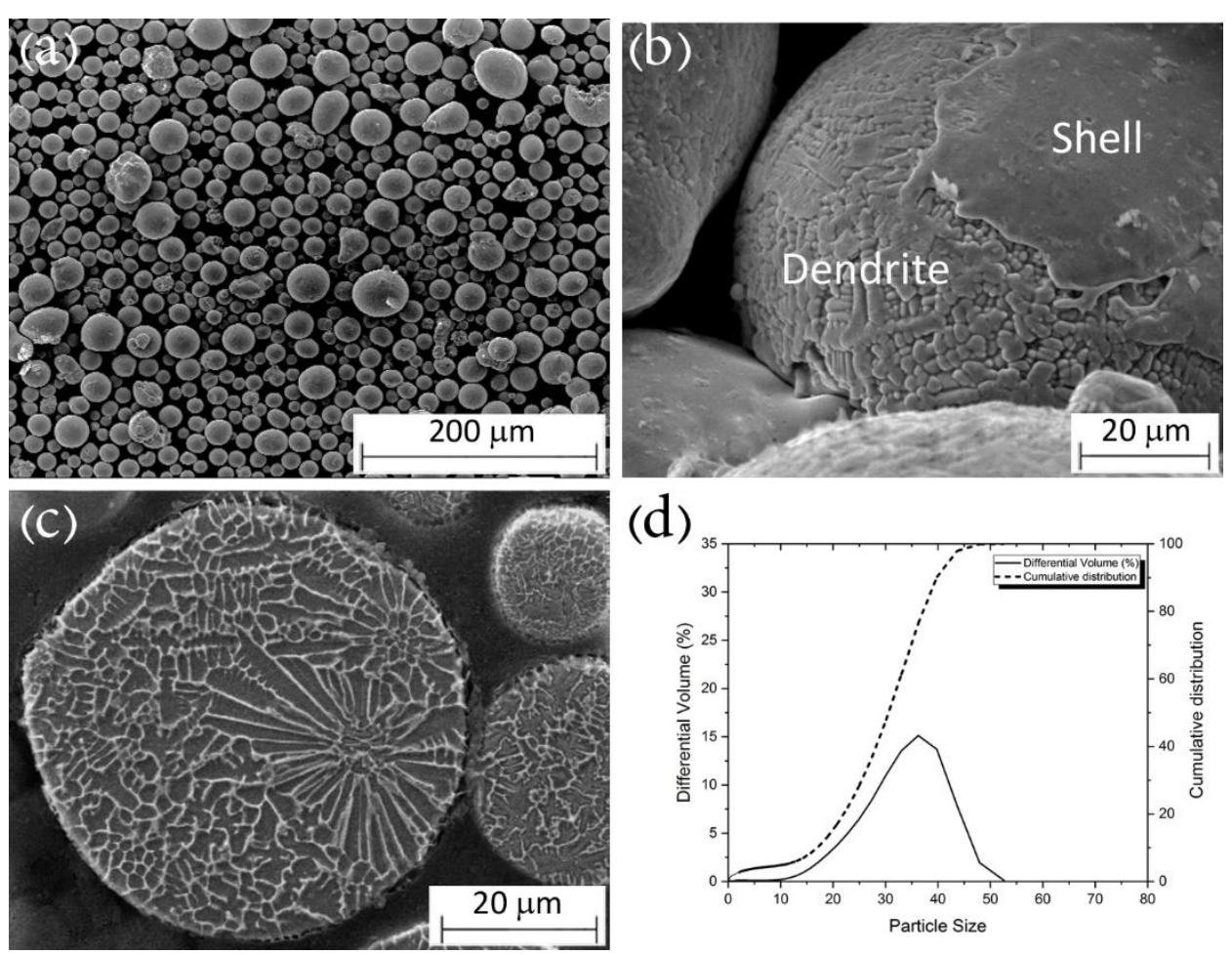

(d)

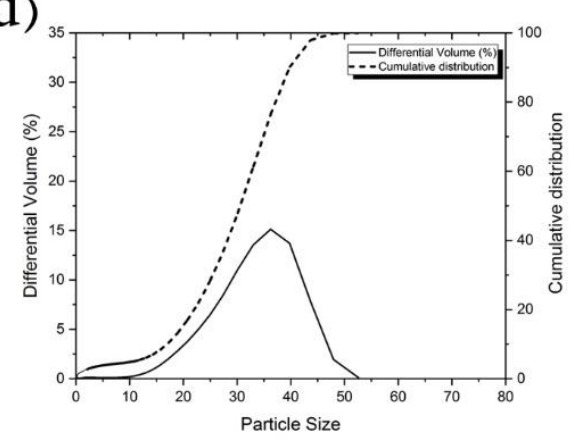




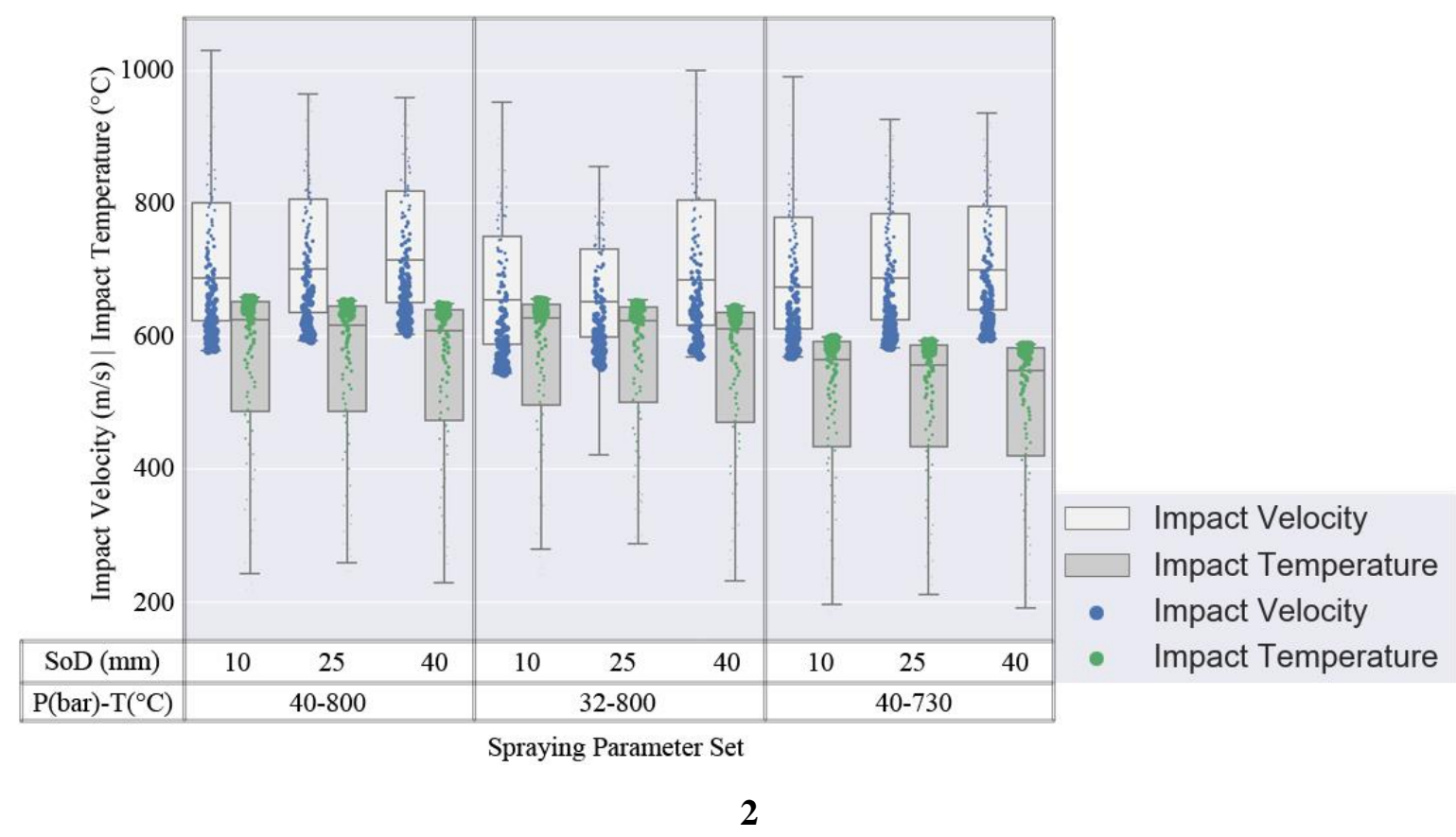



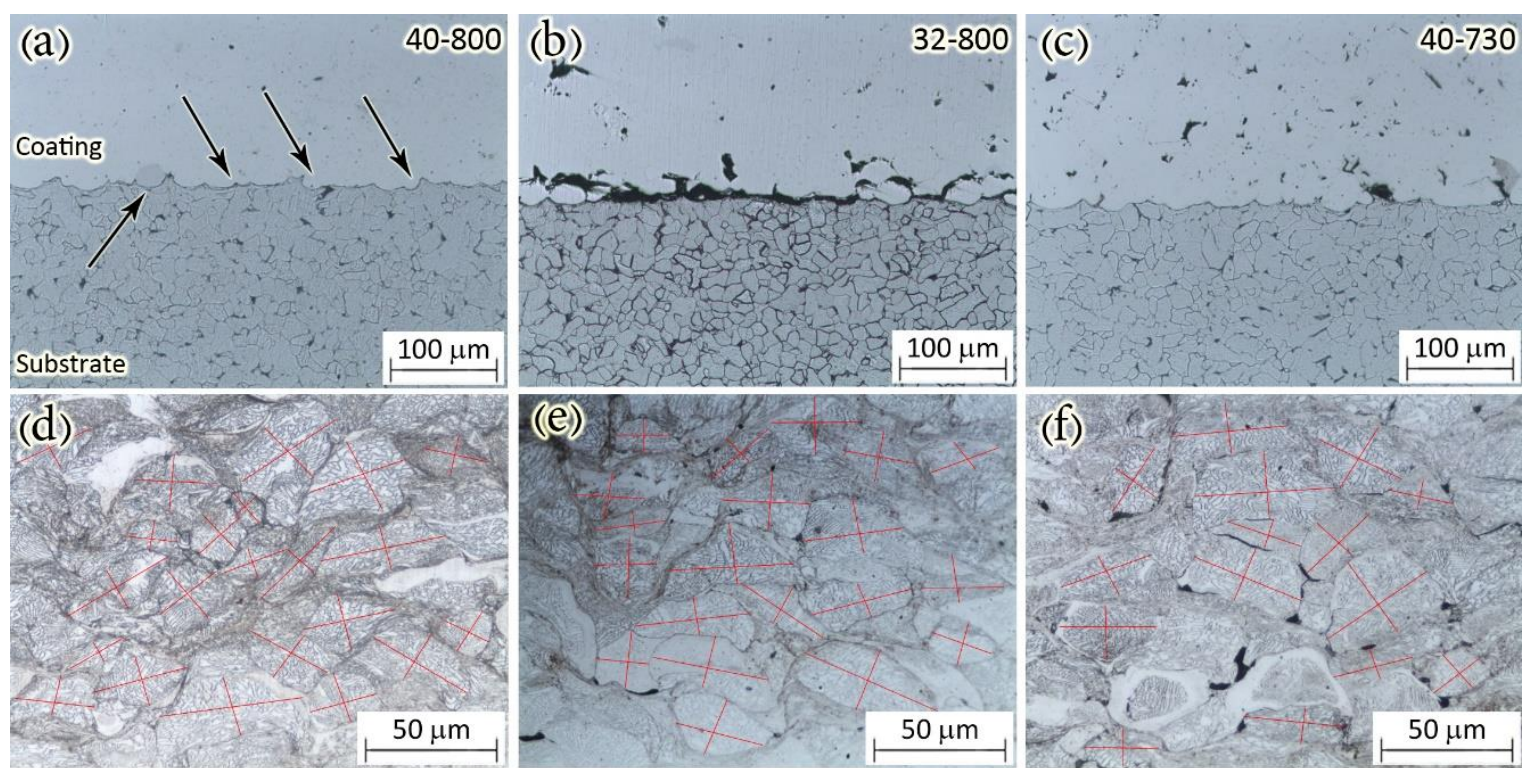


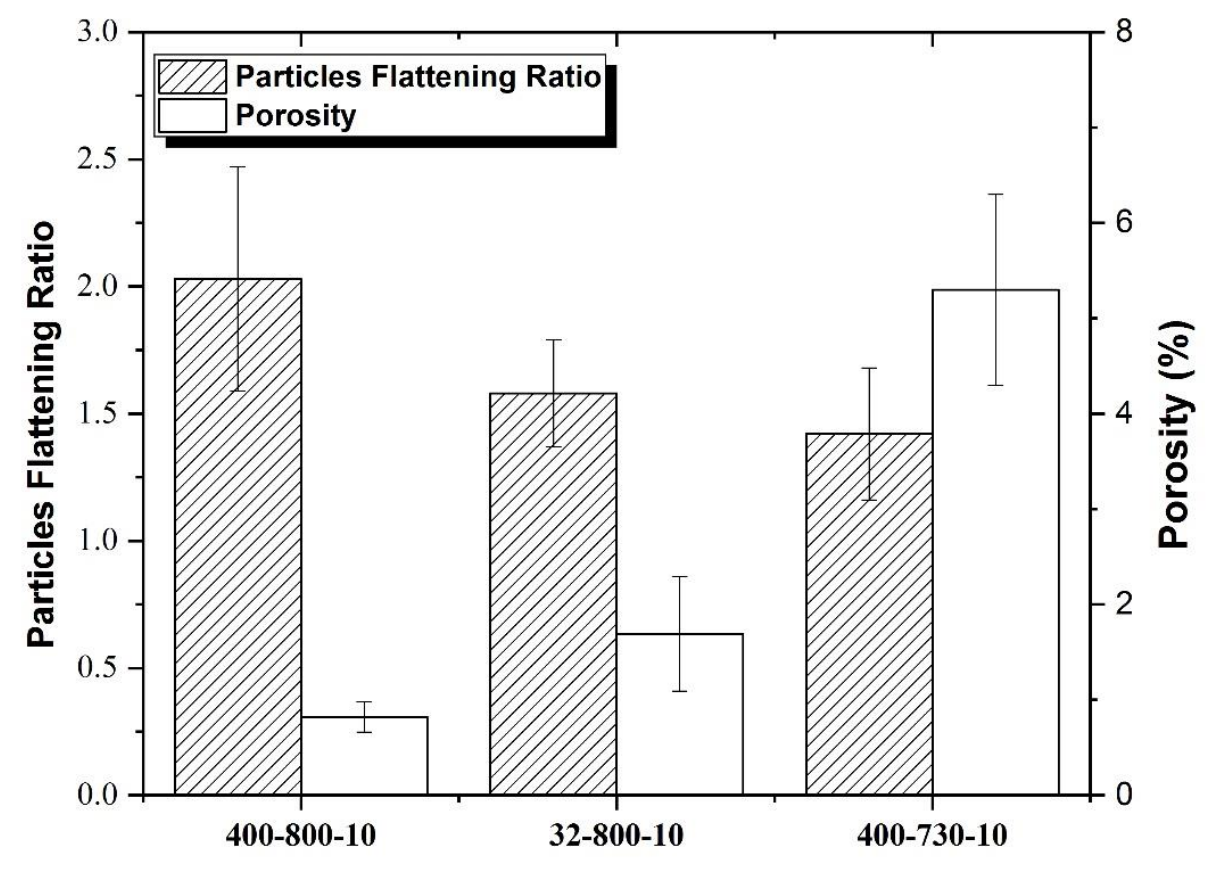

4 


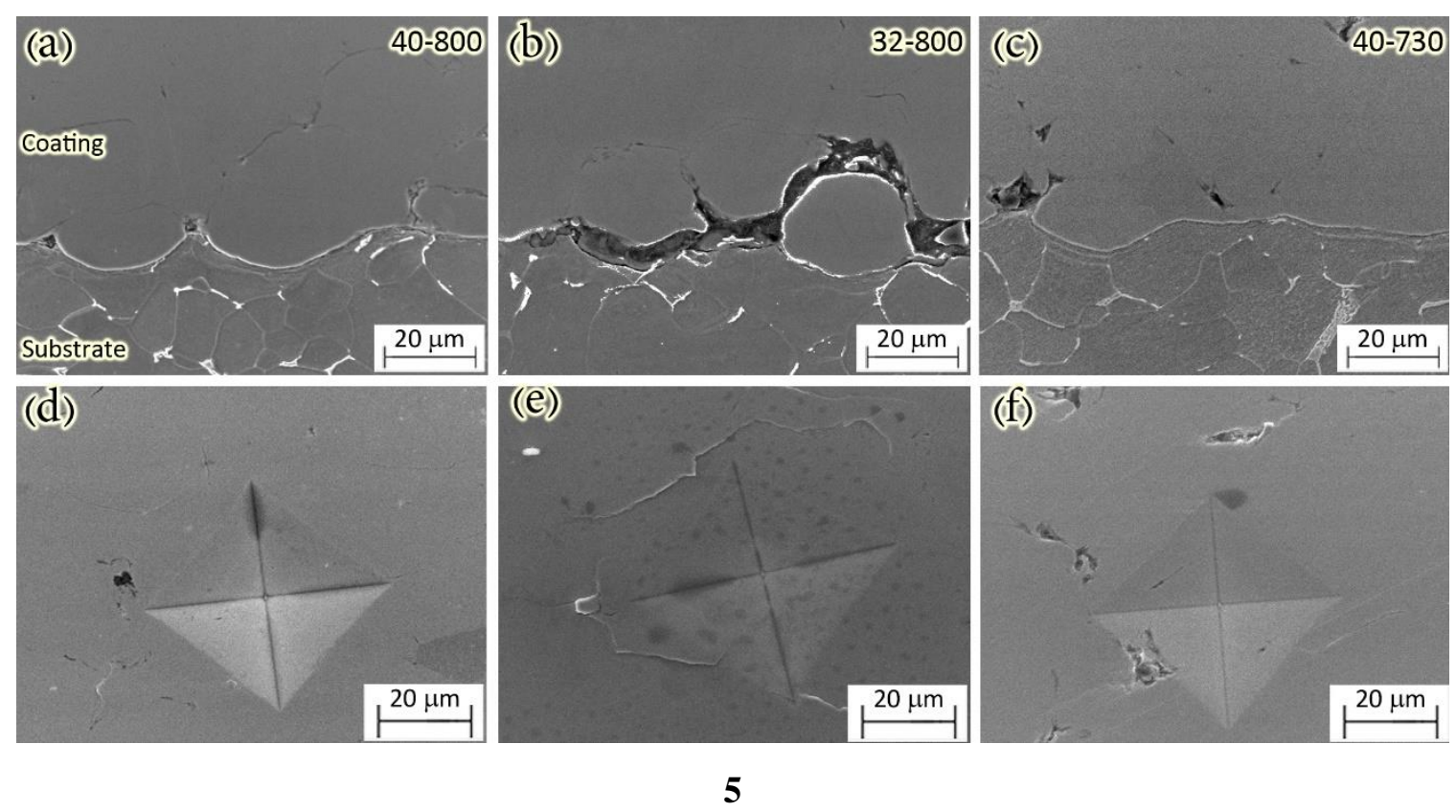



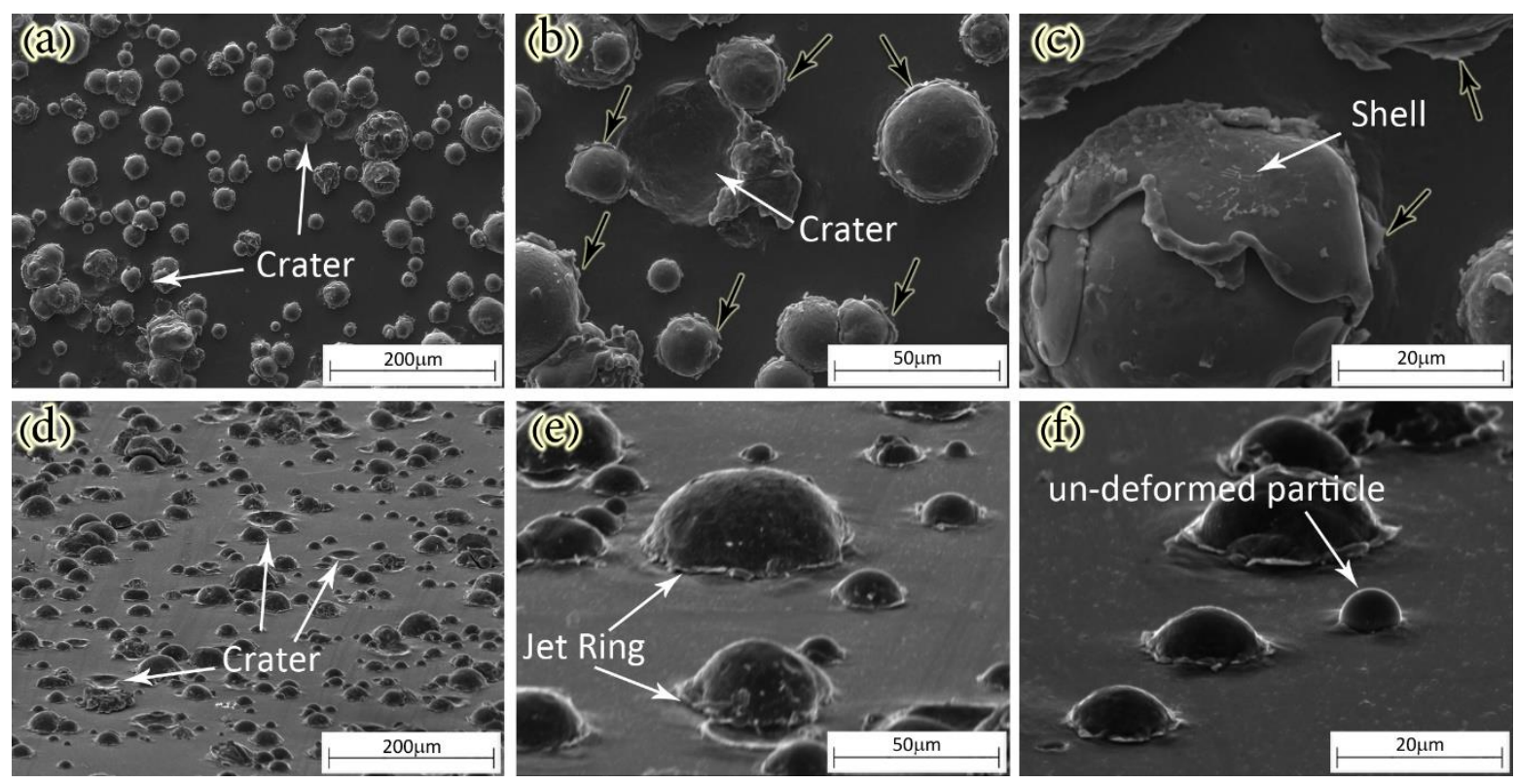

6 


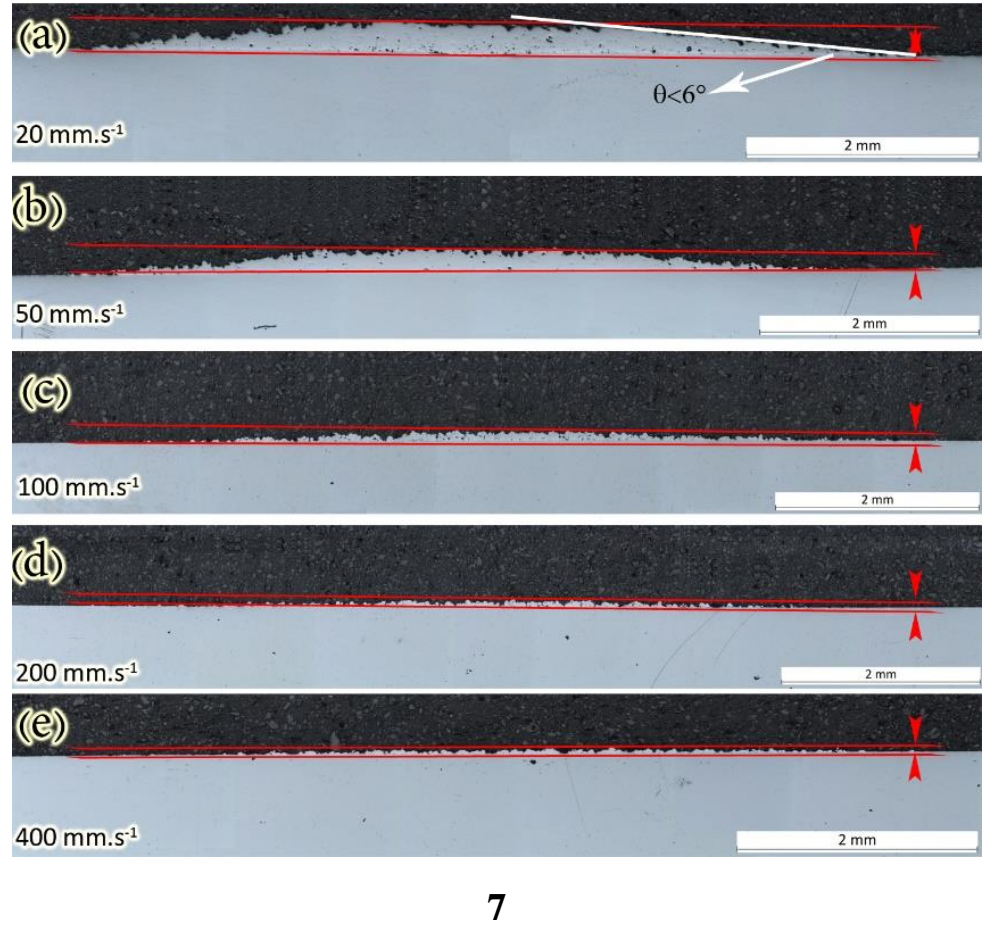




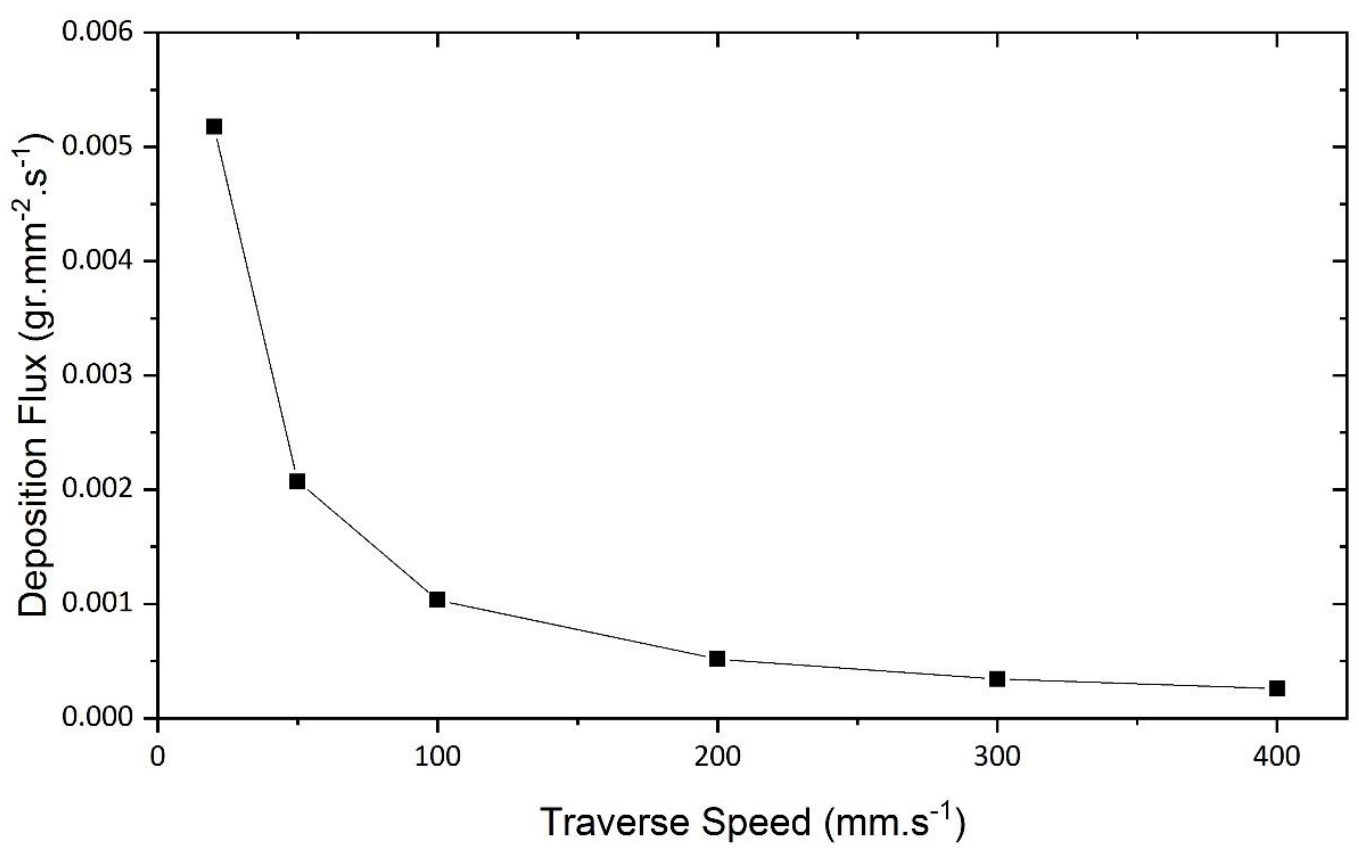

8 


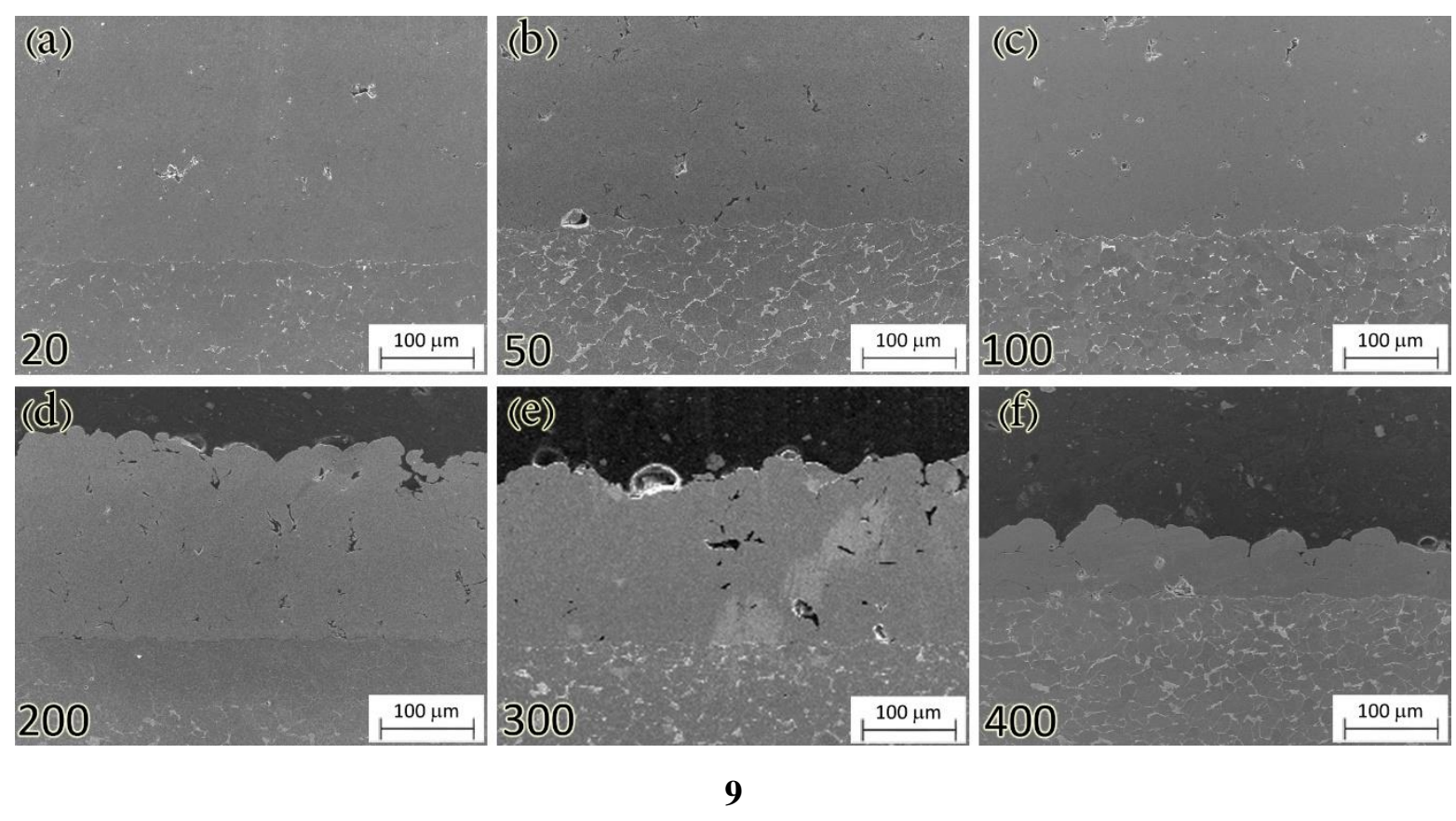




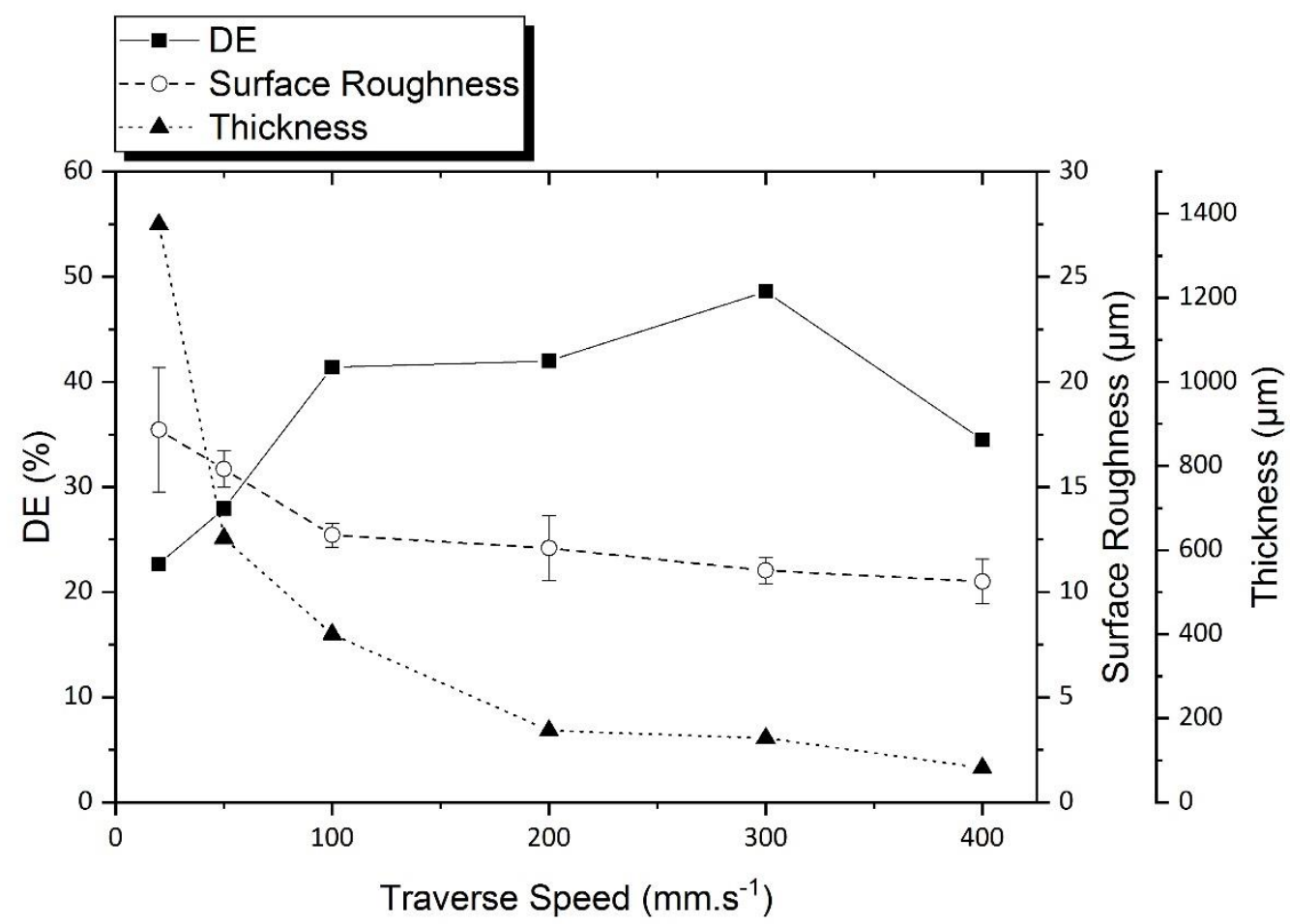

10 
(a)

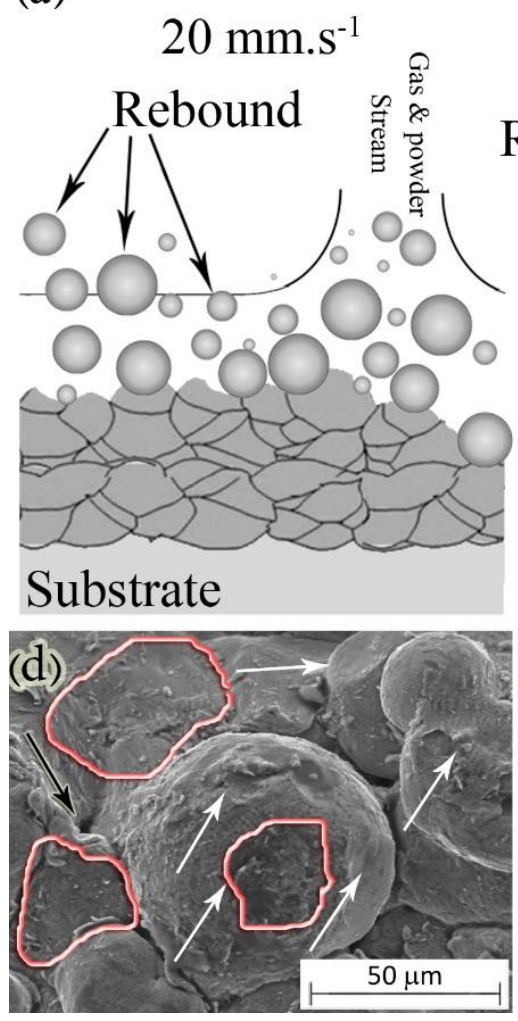

(b)

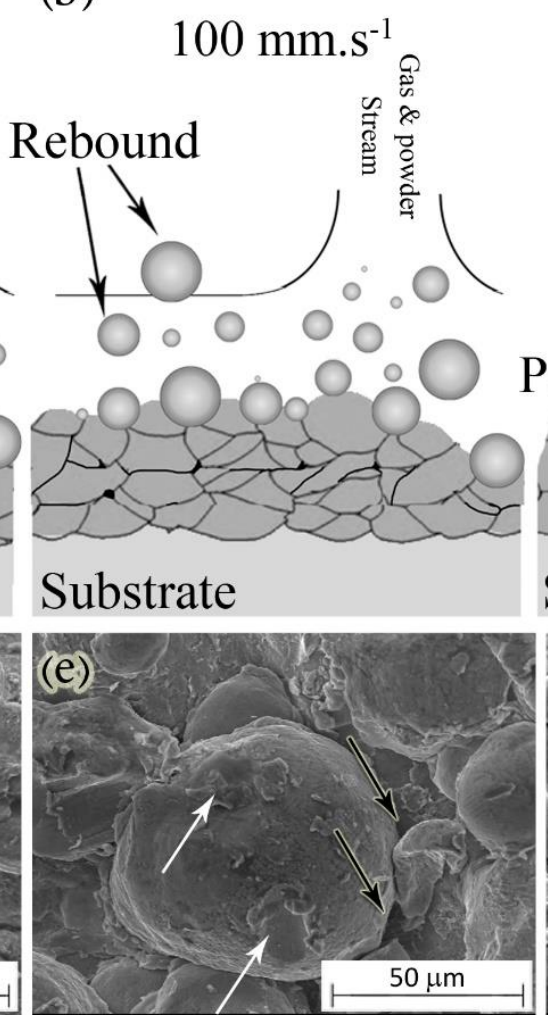

(C)
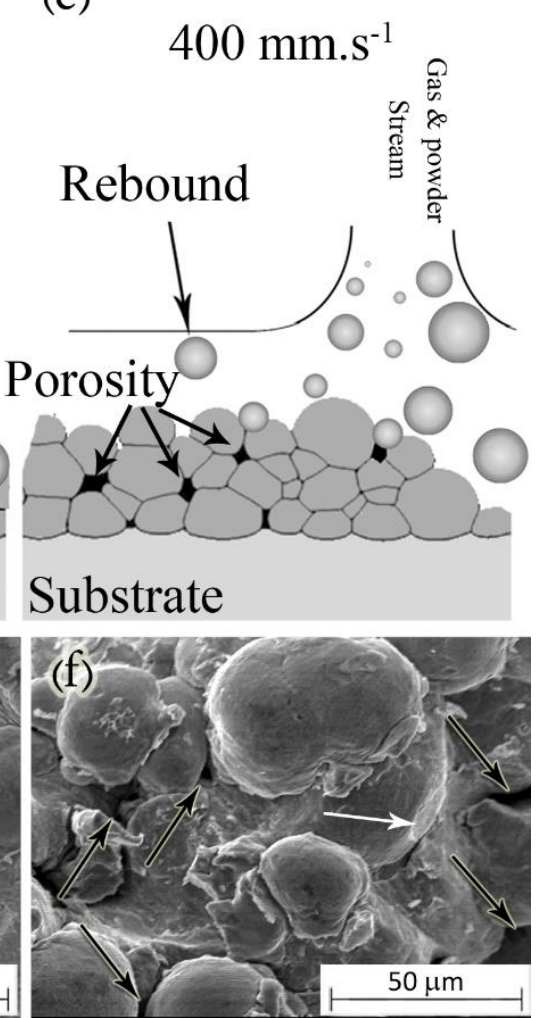


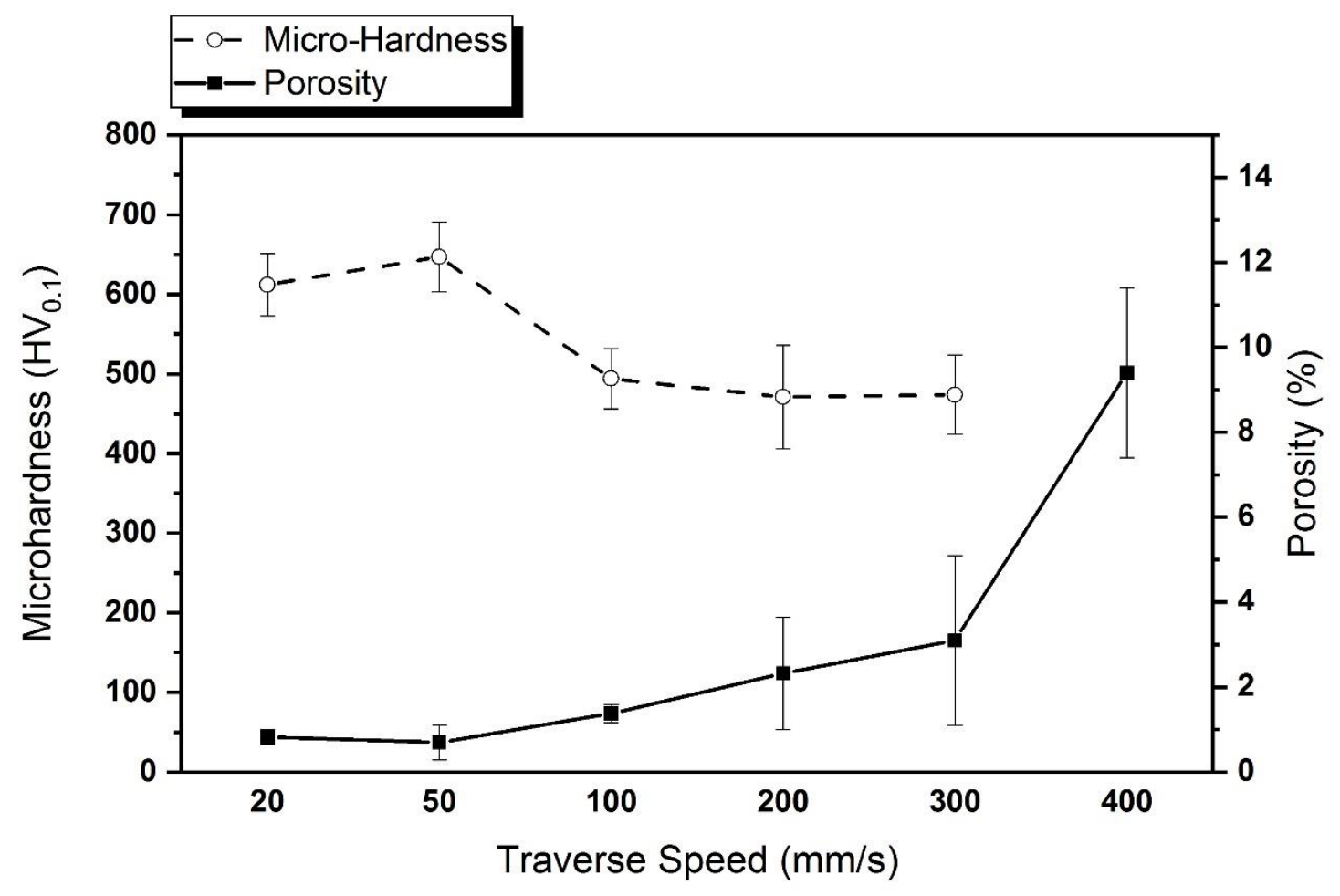

12 